\title{
ELECTROMYOGRAPHIC AND MOTOR CORTICAL RESPONSES TO IMPOSED DISPLACEMENTS OF THE CAT ELBOW: DISPARITIES AND HOMOLOGIES WITH THOSE OF THE PRIMATE WRIST ${ }^{1}$
}

\author{
W. G. TATTON, ${ }^{2}$ A. G. E. NORTH, I. C. BRUCE, AND W. BEDINGHAM \\ Playfair Neuroscience Unit, University of Toronto, Toronto Western Hospital, Toronto, Ontario, Canada M5T 2S8
}

Received December 13, 1982; Revised March 23, 1983; Accepted April 1, 1983

\begin{abstract}
The present "loop analysis" study was undertaken to determine the extent to which the segmented electromyographic (EMG) responses of cat triceps brachii to imposed elbow displacements can be used as a model for those occurring in primate wrist musculature under similar input conditions, since previous workers have assumed homology between the two systems. To this end, the response properties of the EMG peaks and the range of latencies available to a "transcortical loop" were determined in the cat and compared with previous findings from primates.

During chronic experiments, simultaneous recordings were made from motor cortical neurons (MCNs) and triceps EMG in response to step loads imposing flexion on the elbow joint. Subsequent acute experiments in the same animals used intraspinal microstimulation to determine the antidromic latencies to the area of cortex containing responsive MCNs, and orthodromic latencies to triceps EMG activation from the same loci.

Following displacements imposed from flexed initial joint angles, the EMG was segmented into two or more peaks while, from extended angles, a single segment was followed by a "silent interval" and tonic activity. Segmentation into three peaks was only observed under limited conditions.

Peak 1 showed a constant latency ( 8 to $12 \mathrm{msec}$ ) and resembled the primate M1 segment. A "silent interval," not present in primate wrist muscles, followed peak 1 , and its duration varied with the average velocity of the imposed displacement. Peak 2 followed the "silent interval" at variable latency ( 34 to $52 \mathrm{msec}$ ), in contrast to the constant and shorter latency shown by the primate wrist M2. Peak 3 was variable in appearance and latency (69 to 85 msec when distinguishable) and its homologies remain unclear. Peak 1 was monotonically graded with average velocity of displacement, whereas peaks 2 and 3 showed variable responses.

Summation of the afferent and efferent latencies for the "receiving zone" of the motor cortex gave modal values of 19 to 23 and 23 to $27 \mathrm{msec}$ for the expected time of occurrence of EMG activity dependent upon MCNs responding to the imposed displacement as part of a "transcortical loop." This activity would therefore occur during the "silent interval," in contrast to the M2 segment in primate wrist muscles, where MCN activity seems to be appropriately timed to contribute to this segment.

It is concluded that the cat triceps brachii EMG responses, together with the related motor cortical responses to imposed elbow displacements, cannot be utilized as a model to study the mechanisms underlying the longer latency EMG segments observed at the primate wrist.
\end{abstract}

Invasive methods, including lesioning, microstimulation, and single neuron recording techniques, carried out in the cat and in a few subhuman primate species have provided information essential to the understanding of

\footnotetext{
${ }^{2}$ This research was supported by Medical Research Council of Canada Grant MA 5218 to W. G. T. and I. C. B. We wish to thank R. Andrews for modifying the computer programs to the requirements of this work, and M. Hay and J. A. Glen for technical assistance.
}

neural circuits underlying mammalian motor control. In attempts to determine whether similar circuitry operates in man, one approach has been to compare electromyographic (EMG) activity during specific movement paradigms in experimental animals to that in man (see

\footnotetext{
${ }^{2}$ To whom correspondence should be addressed, at Playfair Neuroscience Unit, Toronto Western Hospital, 399 Bathurst Street, Toronto, Ontario, Canada M5T 2S8.
} 
Tatton et al., 1978). The observed EMG patterns are interpreted as reflecting the levels of excitability in specific motoneuron pools and the influences of descending and/or segmental inputs on them.

Using this approach, the segmented bursts of EMG activity in response to imposed perturbations applied across different joints have been examined in man, monkey, and cat (see Hammond, 1954; Tatton et al., 1975; Ghez and Shinoda, 1978, for examples). Yet, despite the fact that apparently identical perturbation paradigms have been used, two interrelated problems raise questions as to whether results obtained by invasive techniques in animals can be used to interpret data obtained by noninvasive methods in man.

First, within a single species, different joint-muscle systems are required to perform repertoires of disparate motor tasks and can encounter dissimilar kinds of environmental input during their normal functioning. Thus, the "weighting" or even the occurrence of a specific neural mechanism might vary widely between different joint-muscle systems in a single species (Lenz et al., 1983a, b). In fact, homology between peripheral and central mechanisms may not even extend to muscles acting across the same joint, as is evident from studies of soleus and tibialis anterior during ankle perturbations in man (Gottlieb and Agarwal, 1980).

Second, the musculoskeletal systems of different species are adapted to perform characteristic motor functions which may utilize unique neuronal circuits, or even the same circuits in various ways to reflexively modify movements in activities as dissimilar as walking, swimming, or flight.

In early studies, arguments based on the latencies of the EMG segments were used to draw inferences as to the likely pathways involved in their generation, especially with regard to the longer latency segment (M2) in the primate distal upper limb and its dependence on the motor cortex. Subsequently, the simple observation of a segmented EMG response has been regarded as sufficient to indicate the presence of common underlying mechanisms, irrespective of species or joint-muscle system.

In the present work, we chose to carry out a "loop analysis" study of the most clearly segmented EMG response reported to date, that of the cat triceps brachii, since it is this system which has been widely quoted as providing definitive evidence against the existence of a "transcortical loop" (Ghez and Shinoda, 1978). We attempt to resolve some of the conflicts in the literature by examining the characteristics of the perturbationevoked segmented EMG responses, the timing of simultaneously recorded motor cortical neuronal responses, and corticospinal conduction times related to triceps brachii in the cat, i.e., define the time constraints imposed on a hypothetical "loop" through the motor cortex. The results are compared with the most intensively studied system to date, that of the primate wrist (the term "primate" in this context rightly includes man).

Our study demonstrates that the EMG responses in these two muscle-joint systems (cat elbow and primate wrist) show major disparities. Specifically, the long latency EMG segments in cat triceps show response properties that differ from those in primate wrist muscles, and the timing of motor cortical neurons' activity recorded simultaneously with the EMG activity is not appropriate to contribute to the cat triceps EMG responses. A preliminary account of some of this work has been presented (North and Tatton, 1980).

\section{Materials and Methods}

Chronic preparation. Seven cats ( 3 to $4 \mathrm{~kg}$ ) were prepared under barbiturate anesthesia. Three stainless steel holding bolts were implanted in the dorsolateral parietal bone. The lateral pericruciate cortex on one side was exposed and covered by a 1-cm-diameter stainless steel well sealed over the craniotomy and adapted to carry the hydraulic microdrive. The vertical axis of the well was oriented approximately parallel to the rostrocaudal angle of the cruciate sulcus. The holding bolts, well, and exposed bone were finally embedded and fixed in dental acrylic (Fig. 1). The animals tolerated the surgery well and were moving and feeding normally $24 \mathrm{hr}$ later.

On the 3rd postoperative day, being either fully awake (three animals), or lightly tranquilized with acepromazine (four animals), the cat was wrapped in a restraining bag and its head was painlessly immobilized by attaching threaded bars to the holding bolts. The shoulder girdle was immobilized by a fitted cast which restricted movement to the elbow joint contralateral to the recording well. With appropriate adjustments for each cat, the center of rotation of the elbow joint was maintained approximately coaxial with the shaft of a precision torque motor (Fig. 1).

The motor, under the control of a PDP $11 / 34$ computer, imposed step loads to flex the elbow. Loads were randomized as to duration (450 to $600 \mathrm{msec}$ ), interval between loads ( 3 to $5 \mathrm{sec}$ ), and sequence of presentation of four specified load magnitudes $(0.04$ to 0.16 newtonmeters, chosen to correspond to those used previously by Ghez and Shinoda, 1978). The motor's time to peak torque was $3.2 \mathrm{msec}$. An infinite resolution potentiometer

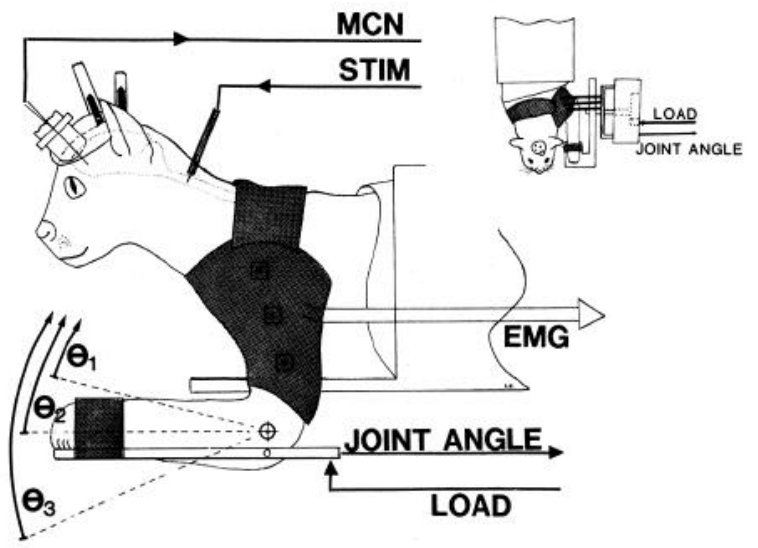

Figure 1. Schematic illustration of experimental arrangements for chronic and acute experiments. In the chronic experiments the activity of motor cortical neurons $(M C N)$ and triceps brachii $(E M G)$, along with joint angle, were recorded in response to imposed loads. In acute experiments, a simulating electrode was manipulated into the cervical spinal cord $(S T I M)$. The inset shows a dorsal view to illustrate the manipulandum. Stabilizing attachments are shaded. $\theta_{1}, \theta_{2}$, and $\theta_{3}$ indicate three initial joint angles. 
attached to the motor's shaft provided a continuous measure of limb position (joint angle). In individual experiments, displacements were imposed from mechanical stops placed at three different initial joint angles $\left(\Theta_{1}, \Theta_{2}\right.$, and $\Theta_{3}$ in Fig. 1), and the manipulandum moved the forelimb into flexion to a padded stop, stretching triceps brachii.

EMG activity was recorded differentially through pairs of $75-\mu \mathrm{m}$ wires insulated to within $2 \mathrm{~mm}$ of the tip, inserted into the long head of triceps brachii. The activity of single motor cortical neurons (MCNs) was recorded using insulated tungsten microelectrodes mounted on a microdrive and micropositioner which allowed penetrations to known depths in a 1-cm grid over the lateral pericruciate cortex (area 4 of Hassler and Muhs-Clement, 1964). Daily 3- to 5-hr recording sessions were carried out over periods of 3 to 6 weeks.

Triceps EMG, MCN activity, joint angle, and motor control pulses were recorded on magnetic tape for subsequent off-line analysis.

Acute preparation. In order to obtain precise measurements of conduction times from motor cortex to triceps brachii, the chronic recording period was terminated by an acute experiment for five of the cats. Following anesthesia with chloralose:urethane $(10 \%: 1 \%, 7 \mathrm{ml} / \mathrm{kg})$ and laminectomies from $\mathrm{C} 2$ to $\mathrm{C} 6$, the animal was suspended in a spinal recording frame and the exposed cord was maintained in a warmed pool of mineral oil (Fig. 1).

Procedures for recording from single MCNs and triceps EMG during torque motor-imposed elbow displacements were carried out as for the chronic experiments. Under microscopic control, a concentric stimulating electrode (diameter, $150 \mu \mathrm{m}$ ) was micromanipulated in a grid of penetrations in the contralateral $\mathrm{C} 2$ to $\mathrm{C} 3$ spinal cord for antidromic identification of simultaneously recorded MCNs. All but the most ventral areas of the hemichord were explored. Trains of $0.1-\mathrm{msec}$ pulses at 50 to $250 \mathrm{~Hz}$ with current intensities up to $50 \mu \mathrm{A}$ were delivered. Antidromic activation of MCNs was defined by: (1) fixed latency, varying less than $50 \mu$ sec: (2) one-for-one following up to $150 \mathrm{~Hz}$; and (3) collision with spontaneously occurring action potentials. Simultaneously, the same intraspinal microstimulation method was used to determine times from $\mathrm{C} 2$ or $\mathrm{C} 3$ stimulation to the onset of triceps EMG activation.

At the end of each acute experiment, electrolytic lesions were made in the motor cortex and spinal cord to facilitate subsequent reconstruction of electrode tracks. The animals were then killed by anesthetic overdose and perfused with isotonic saline followed by $10 \%$ buffered formalin. Serial frozen sections through pericruciate cortex and cervical cord were cut at $50-\mu \mathrm{m}$ intervals and stained with Luxol fast blue and cresyl violet for identification of electrode positions.

Analysis. Analysis programs separated the randomly sequenced loads by magnitude. A typical sequence of 10 elbow movements imposed by a single load magnitude ( 0.16 newton-meter) is shown in Figure $2 A$. Average joint angle and average rectified EMG activity were constructed for each load magnitude (Fig. 3). Statistical subroutines calculated mean background (prestimulus) EMG activity (designated $U_{b}$, Fig. $2 D$ ), the level $3 S D$

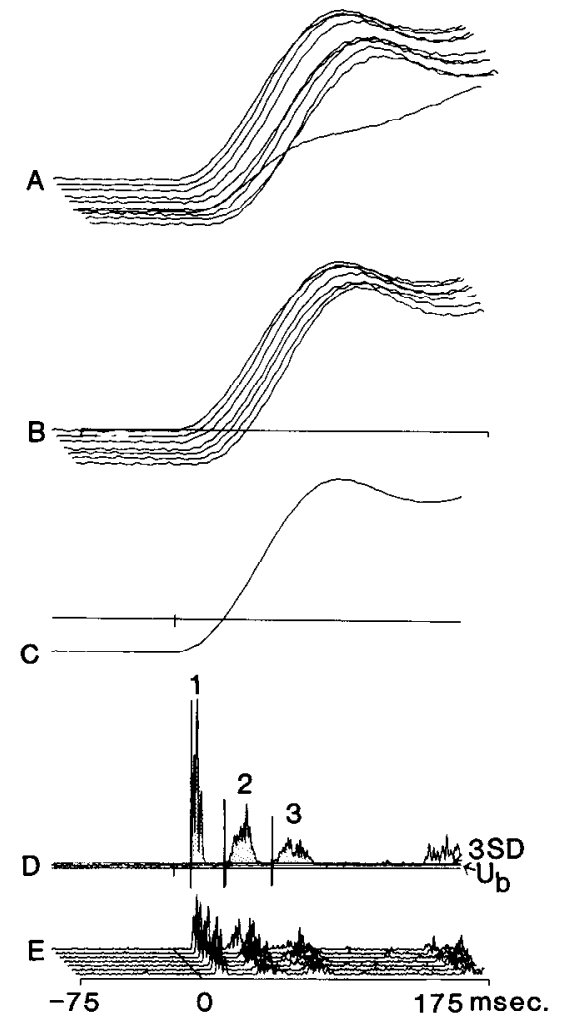

Figure 2. Example of analysis techniques. $A$, Ten joint angle traces for a single load magnitude extracted from the random load sequence. Note that three of the imposed movements did not follow the normal trajectory. $B$, Those movements during which the cat resisted the imposed displacement (the third, fourth and sixth trials) were deleted, providing an average joint angle trajectory $(C)$ and the corresponding average rectified EMG $(D)$. The representative nature of the average was confirmed by observation of the single events from which it was constructed $(E)$. $D$ further illustrates the methods of latency and amplitude measurement from EMG responses. Mean background EMG level $\left(U_{b}\right)$ was calculated for the 75 msec preced ing the onset of the movement, and a line defining 3 standard deviations above the mean $(3 S D)$ was calculated. The points at which the EMG levels exceeded the 3SD level $(1,2$, and 3$)$ were used to measure the onset latencies. The areas under the curve above mean background activity provide the amplitude values for each response peak as millivolts per millisecond per presentation.

above mean background, and the areas above mean background activity encompassed by the response peaks. The mean background activity was calculated from the average EMG level during the steady-state 75 -msec control period immediately preceding the onset of the step load, when the limb had returned to the stationary rest position after the previous trial (statistical criteria are illustrated and defined in Fig. 2D). Latencies were measured directly from the average data as the time between the onset of the motor control signal and the points at which the EMG activity exceeded the 3 SD level illustrated in Fig. $2 D)$.

Unexpected variations in joint stiffness due to the cats' voluntary activity were found in some trials to result in different displacements being evoked by loads of the same magnitude. During data analysis, those individual 
trials in which (1) the imposed displacements varied from a fixed duration and amplitude and (2) voluntary movements preceded the onset of the imposed perturbation were eliminated from the construction of average EMG responses (note the trials eliminated from those in Fig. $2 A$ leave those in Fig. $2 B$ and the corresponding EMG responses in Fig. $2 E$, which were used to construct the average records in Fig. 2, $C$ and $D$ ). Thus the averages for both the imposed movements and the EMG activity were representative of the individual trials used for their construction. Finally, in order to provide a more reliable index of the imposed movement than of load, and recognizing that the step load-imposed movements follow a sigmoidal trajectory, the average velocity of the movement was calculated from the beginning of the movement to the point at which the limb movement reached its maximum extent, and these values were used to construct the "gain," latency, and duration plots of Figures 5 and 6.

Simultaneously recorded single response trials for MCNs which corresponded to the selected EMG trials were used to construct average response histograms (Fig. 8, $C$ and $D$ ). Latencies for MCN responses were measured
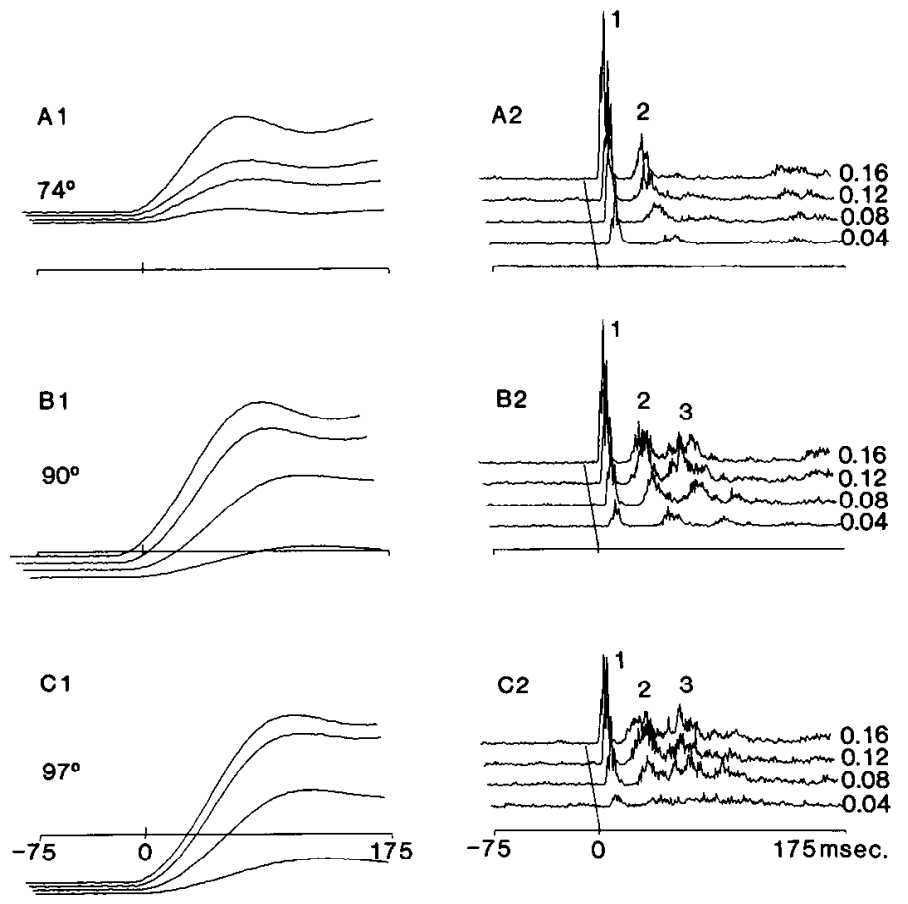

Figure 3. Examples of averaged data from which triceps EMG latency and amplitude values were obtained. These data are from a single experiment in which randomized load displacements were imposed at three different initial joint angles; they illustrate typical responses from initially flexed positions. (Initial joint angle is measured between distal and proximal forelimb, so that $90^{\circ}$ is neutral, less than $90^{\circ}$ indicates flexion, and greater than $90^{\circ}$ indicates extension. All perturbations were in the flexion direction, stretching triceps brachii.) Each panel in the left column shows the average limb displacement produced by each of four imposed loads at initial joint angles of $74^{\circ}(A 1)$, $90^{\circ}(B 1)$, and $97^{\circ}(C 1)$. The corresponding triceps EMG averages are shown in each panel of the right column $(A 2, B 2, C 2)$, with their imposed load values in newton-meters to the right of each average. The values for average velocities, EMG amplitudes, and latencies are given in Figure 5.

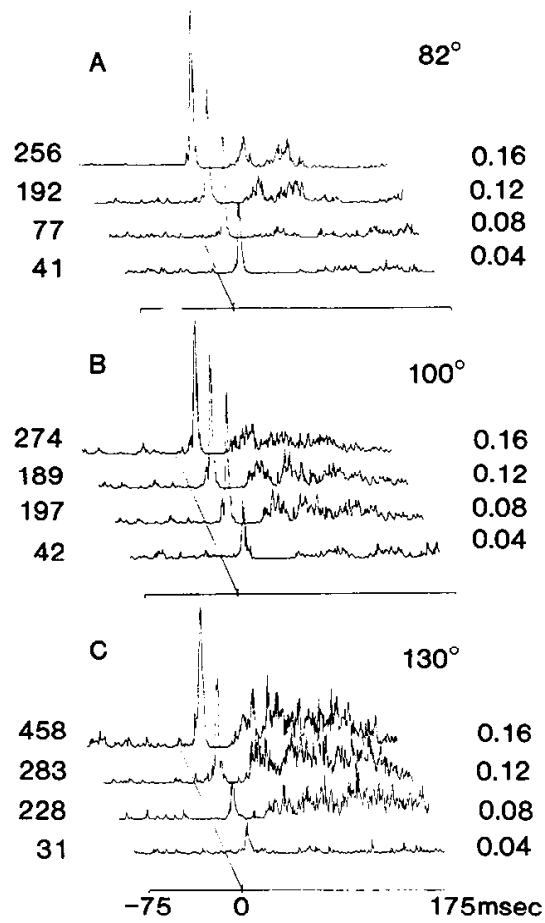

Figure 4. An example of averaged EMG responses from extended initial joint angles of $82^{\circ}(A), 100^{\circ}(B)$, and $130^{\circ}(C)$ in one experimental run. The numbers to the left of each trace show the average velocities of displacement (degrees per second) produced by the imposed loads (newton-meters) shown on the right of each trace.

using mean background activity and $3 \mathrm{SD}$ criteria as described for the EMG averages above.

\section{Results}

Latencies of EMG responses to imposed displacements. We found that the EMG responses to imposed displacements were not invariably segmented. Three peaks could be distinguished when the displacements were imposed from flexed initial joint angles (less than 100 to $110^{\circ}$ ), as illustrated in Figures $3, B$ and $C$, and $4 A$, even at low average velocities of displacement (see fourth trace, Fig. $3 B 2$ ). However, only peak 1 could be readily identified in most cases where the initial joint angle was greater than 100 to $110^{\circ}$, further peaks being indistinguishable from the tonic activity (Fig. $4, B$ and $C$ ). Finally, shorter durations could result in only two peaks (Fig. $3 A$ ), whereas more prolonged movements could evoke a fourth peak (Fig. 3B2, third trace). The variability in the movements produced by the same loads (compare velocity and load values in Fig. 4) imposed at different initial angles demonstrates that simply considering load as the input variable can be misleading.

In order to determine whether the peaks were generated by relatively "simple" reflex circuitry, as in a monoor polysynaptic excitatory circuit of high synaptic security, where latency would be largely determined by the time around the "loop," latencies were measured for a range of average velocities from different initial joint angles. Typical effects of average velocity and initial joint angle on latency derived from data such as that illustrated in Figure 3 are plotted in Figure 5A, constructed for three initial joint angles and four average 


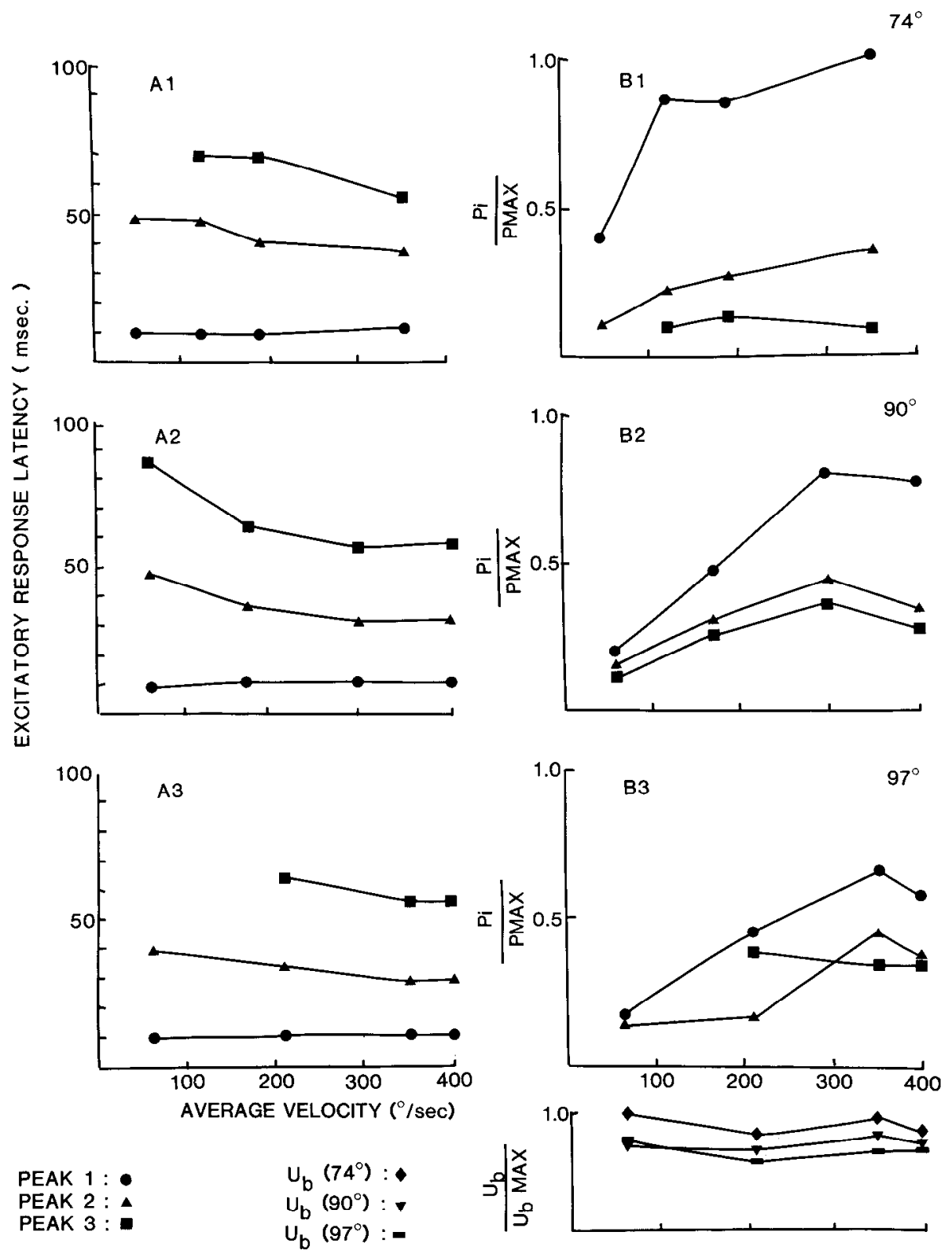

Figure 5. Relationships between imposed load magnitude and EMG latency for the first (circles), second (triangles), and third (squares) EMG components in triceps brachii for a typical experiment. The left panels show the effects of average velocity of the imposed movement (degrees per second) on excitatory response latency at initial joint angles of $74^{\circ}(A 1), 90^{\circ}(A 2)$, and $97^{\circ}(A 3)$. The right panels show the corresponding effects on the amplitudes of the EMG components. The area above the mean background level for a given average response peak in millivolts per millisecond $(P i)$ was normalized to the area of the largest peak (PMAX) on the ordinate of each panel $(B 1, B 2, B 3)$. The lowest panel shows the average background EMG activity preceding the displacements $\left(U_{b}\right)$ normalized against the maximum background activity $\left(U_{b} M A X\right)$ for each average velocity at the different initial joint angles.

velocities. Latencies for peak 1 ranged between 8 and 12 msec in the seven animals but were constant to within $\pm 1 \mathrm{msec}$ in a single animal. As shown by the circles in Figure $5 \mathrm{~A}$, peak 1 latency was not affected by initial joint angle or average velocity, thus exhibiting the characteristics expected of a "simple" reflex.

In marked contrast, the latencies of peaks 2 and 3 (triangles and squares, respectively, in Fig. 5A) consistently decreased as the average velocity increased. The influence of initial joint, angle was less marked, but peak
2 and peak 3 latencies always increased for a given velocity as the initial joint angle became more flexed. The latency shifts observed for peak 2 usually approached $20 \mathrm{msec}$, while those of peak 3 were even greater, often exceeding $30 \mathrm{msec}$ (e.g., squares in Fig. $5 A 2$ ), suggesting that other factors, rather than just a "simple" reflex, could play a role in the generation of the longer latency EMG segments.

The ranges of onset latencies for the three peaks (at the maximum imposed load of 0.16 newton-meter corre- 
sponding to an average velocity of $400^{\circ} / \mathrm{sec}$, pooled from all animals) were 8 to 12,34 to 52 , and 60 to 85 msec for peaks 1,2 , and 3 , respectively.

Amplitudes of EMG responses to imposed displacements. An indicator of the number of motoneurons recruited during the EMG responses can be provided by constructing an input/output plot, input being a parameter of the imposed perturbation, such as average velocity, and output the average amplitude of the EMG peak. From averaged data such as those illustrated in Figures 3 and 4, the input/output relationships for each peak at each initial joint angle were calculated (Fig. $5 B$ ). The input was expressed as average velocity of displacement (degrees per second), and the output was calculated by normalizing the integrated EMG activity in each peak $(P i)$ above the mean background activity $\left(U_{b}\right)$ against the maximum activity evoked $(P M A X)$ in each experimental session (see Tatton and Bawa, 1979, for the rationale of this method).

At each initial joint angle, peak 1 showed a monotonic increase, indicating enhanced excitability in the triceps motoneuron pool, which often appeared to reach a maximum at the higher velocities (Fig. $5 B$, circles). Peak 2 also showed a graded increase with increasing average velocity, although usually with a lesser slope than peak 1 (Fig. 5B, triangles). In contrast, peak 3 (which most often could not be measured at the lower average velocities) only showed a graded response at the neutral initial joint angle (Fig. 5B2, squares), while in this case, maintaining a relatively low constant level when perturbed from the flexed initial joint angle (Fig. 5B1) and a more elevated level from the extended initial joint angle.

Background EMG activity preceding the onset of the perturbations $\left(U_{b} / U_{b} M A X\right)$ remained relatively constant throughout each experiment for a given joint angle (see lowest panel in Fig. $5 B$ ). Relative constancy of this measure is crucial to the interpretation of input/output relations, since the level of EMG activity (i.e., background motoneuron activity) immediately preceding the load onset strongly influences the amplitude of the response peaks (Tatton and Bawa, 1979; Bedingham and Tatton, 1981). Thus the input/output curves for peaks 1 and 2 appeared monotonic, while peak 3 (when it was sufficiently prominent to allow measurement) was questionably related to the average velocity of displacement at different initial joint angles.

The "silent interval." A period of decreased motoneuron activity, during which average EMG levels remained below mean background levels, has been termed the "silent interval," and it consistently followed the first EMG peak in triceps (see Figs. 2 to 4, and 9). Since, during this period, EMG activity could decrease to undetectable levels even in the averaged data (see Fig. $8 E$ ), the magnitude of the decrease in activity could not be quantified directly. Alternatively, the duration of the silent interval, i.e., the length of time during which the EMG activity remained below the mean background level, was taken as an index.

The duration of this interval showed a monotonic decrease with increasing average velocity of displacement independent of initial joint angle. A typical result is illustrated in Figure 6, although it must be noted that the absolute values for the duration of the interval varied among different animals. The decreasing duration of the silent interval with velocity reached an asymptotic value of $5 \mathrm{msec}$ at an initial velocity of $300^{\circ} / \mathrm{sec}$ (Fig. 6, dashed line). The relationship of silent period duration to the size of peak 1 was not clear, although there may be a trend toward an inverse relationship (Fig. 6, inset) in this example.

In order to further determine whether the silent interval was related to the preceding motoneuron activity during peak 1, large numbers of individual trials were examined for the infrequent cases in which the first peak of EMG activity failed to occur in response to displacements imposed by a maximal load that usually resulted in a well defined first peak. This procedure is illustrated for a sequence of 400 trials in Figure 7. A raster of seven randomly selected trials (Fig. $7 C$ ) showed the typical EMG response peaks on the average (Fig. $7 B$ ) to a flexion displacement (Fig. $7 A$ ). From the 400-trial sequence, the only seven cases where a typical displacement was imposed (Fig. $7 D$ ), but the first response was greatly attenuated or absent (Fig. $7 F$ ), were rastered and averaged. Both the individual trials and the averaged EMG (Fig. $7 E$ ) lack a period of decreased EMG activity corresponding to the silent interval.

Thus the "silent interval" consistently follows and does not occur independently of peak 1 . However, graphic examination of their relationship (illustrated in the inset of Fig. 6) fails to reveal a single monotonic relationship between the magnitude of peak 1 and the duration of the silent interval which would be expected if the magnitude of peak 1 was the only variable that directly determined the duration of the interval. Thus, a number of different interacting factors which include the average velocity and the magnitude of the motoneuron activity during peak 1 may underlie the "silent interval."

$M C N$ responses to imposed displacements and their

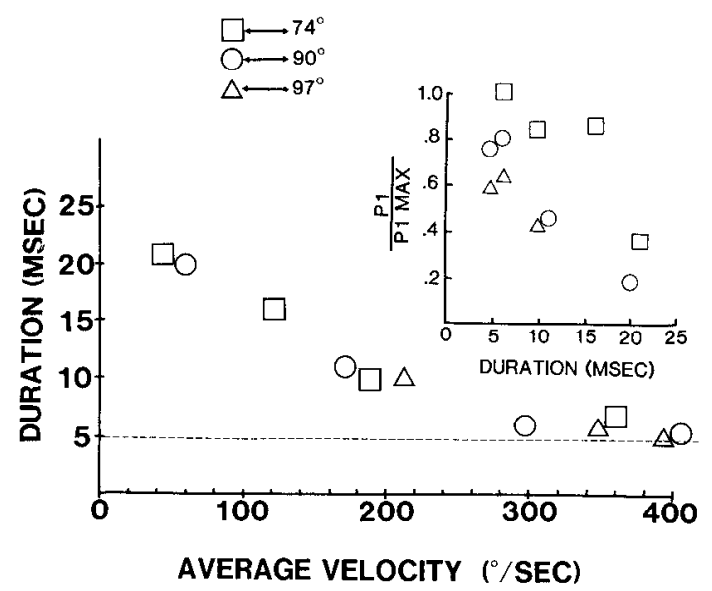

Figure 6. Duration of the "silent interval" (ordinate) following peak 1 plotted against the average velocity of imposed movement ( $a b s c i s s u$ ) for three initial joint angles in one animal. Note that duration decreases linearly with increasing average velocity, reaching an asymptotic value of about 5 msec (dashed line) at $300^{\circ} / \mathrm{sec}$. Inset, Relationship between normalized peak 1 amplitude (P1/P1 MAX) and the duration of the "silent interval." $\square, O, \triangle$, initial joint angles of $74^{\circ}, 90^{\circ}$, and $97^{\circ}$, respectively. 

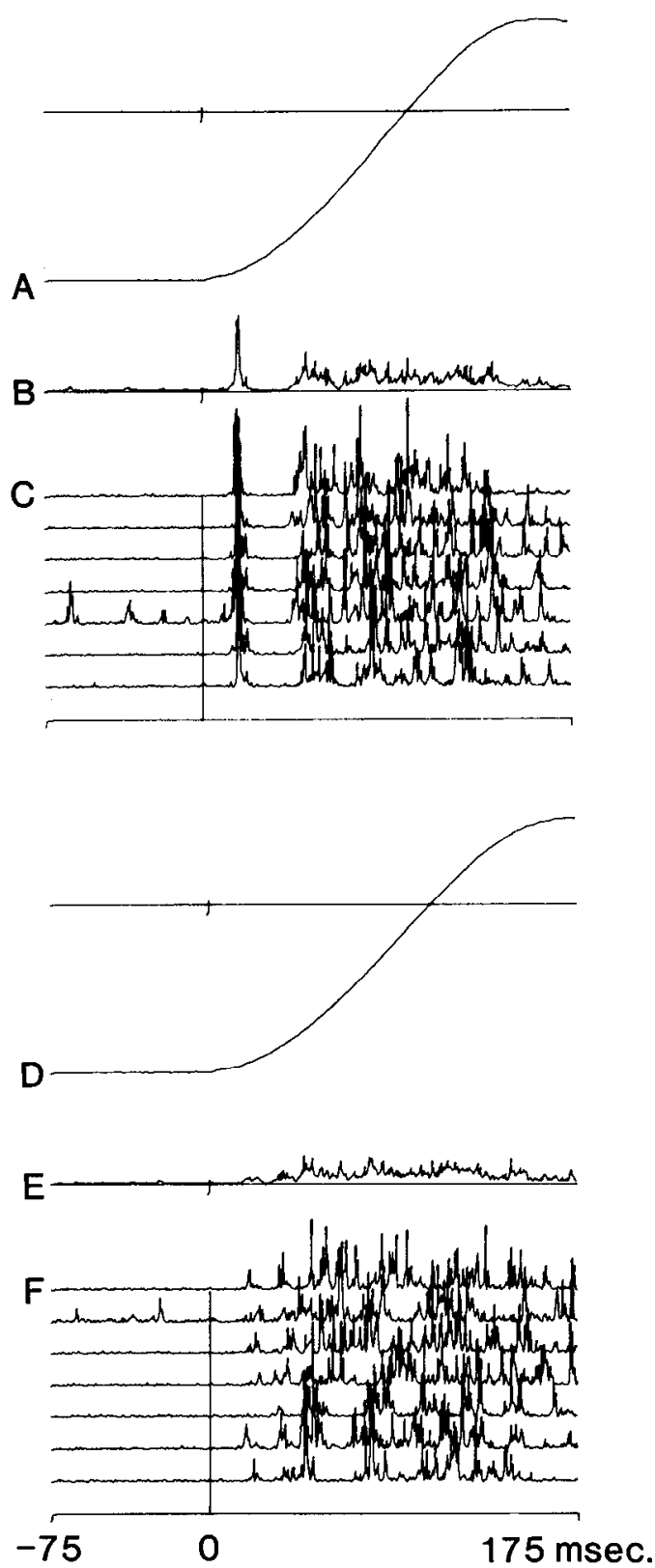

Figure 7. Dependence of "silent interval" on peak 1. The upper panels show the average displacement $(A)$, the average triceps EMG response $(B)$, and a raster of the traces making up the average $(C)$. Note the distinct "silent interval" following peak 1 . In the lower panels are shown the average displacement $(D)$, EMG response $(E)$, and individual trials $(F)$ in which peak 1 was small or absent. Note the absence of decreased EMG activity over the time at which the "silent interval" is normally expressed.

temporal relations to the EMG responses. Simultaneously with the EMG recordings described above, MCN activity was recorded and analyzed according to the same latency criteria described above (see example in Fig. $8 C$ ). By mapping a two-dimensional grid on the pericruciate cortex, a "receiving zone" in area 4 containing MCNs responding to the imposed displacements was delineated during the first 2 to 3 recording days in each cat. The receiving zone was located in an approximately $2-\mathrm{mm}$ wide strip, including both the anterior and posterior banks of the cruciate sulcus, and continued approximately 4 to $6 \mathrm{~mm}$ into its depths adjacent to the lateral end of the sulcus (A. G. E. North, W. G. Tatton, I. C. Bruce, and W. Bedingham, manuscript in preparation).

MCNs in this zone showed excitatory peaks in their average response hislograms at onset latencies ranging between 10 and $28 \mathrm{msec}$ following the beginning of the torque pulse (Fig. 8). Average response histograms showing an initial excitatory peak (responses showing initial decreased firing probability followed by excitation were excluded) provided the minimum and maximum afferent conduction times to the "receiving zone" from forelimb mechanoreceptors activated by the imposed displacements (see Fig. 3 in Bruce and Tatton, 1980). For any single $\mathrm{MCN}$, onset latency was constant within $\pm 2 \mathrm{msec}$ at all initial joint angles tested and over the entire range of imposed loads. The population of $93 \mathrm{MCNs}$ excited by the perturbations showed two distinct modal latencies of 14 to 16 and 18 to 20 msec as illustrated in Figure $9 A$ (of the MCNs analyzed, 417 were inhibited or were unresponsive to the perturbations).

The values for conduction time from the "receiving zone" to triceps activation were then determined. During acute experiments in the same animals, the antidromic

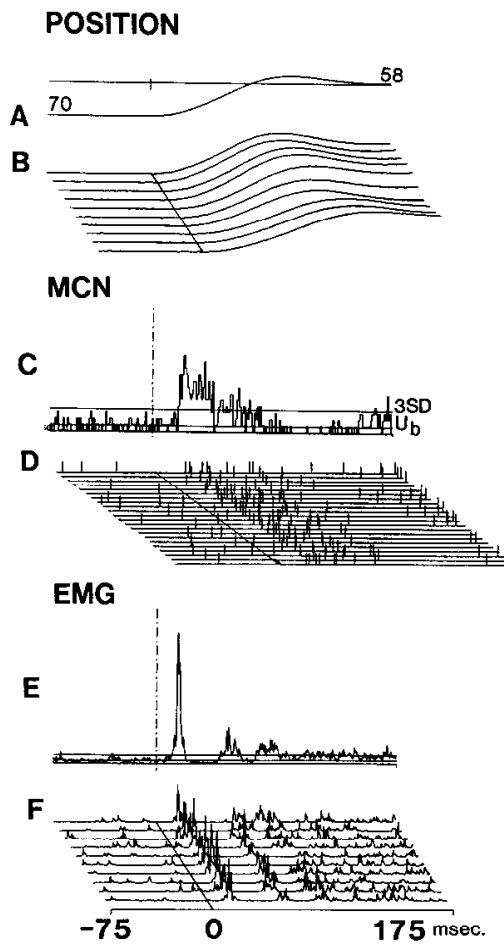

Figure 8. Example of average responses and their constituent rasters obtained from simultaneous recordings of MCN and triceps EMG activity. Upper traces: Average displacement flexing the forelimb from an initial joint angle of $70^{\circ}$ to $58^{\circ}(A)$, and a sample of individual position traces from which it was constructed $(B)$. Middle traces: $C$, Average response histogram showing mean background activity $\left(U_{b}\right)$ and the 3 standard deviations level ( $3 S D)$ from which excitatory response latencies were measured. $D$, A sample of single trials (vertical deflections indicate the occurrence of a spike in a single MCN) comprising the histogram. Lower traces: Corresponding average EMG response $(E)$ and a sample of individual trials $(F)$ recorded from triceps brachii. Zero time $=$ onset of motor pulse. 
A

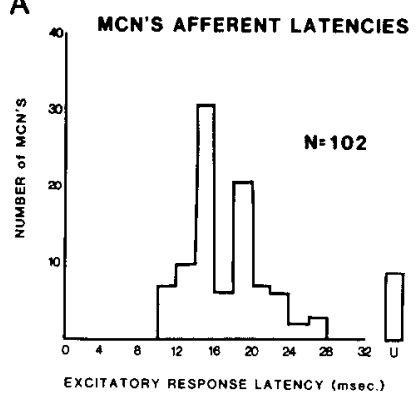

$B$

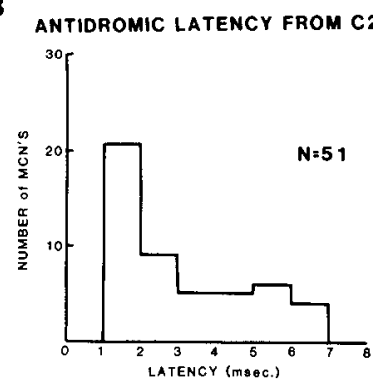

should be borne in mind that the intraspinal stimulation method excites axons at $\mathrm{C} 2$ to $\mathrm{C} 3$ which project to the triceps motoneurons at $\mathrm{C} 6$ to $\mathrm{C} 8$, and that current spread to adjacent descending axons at $\mathrm{C} 2$ to $\mathrm{C} 3$ may be responsible for later EMG activity (see Alstermark et al., 1981, for a description of pathways and their roles in forelimb movements). Thus the minimum and maximum conduction times for activity in the motor cortical "receiving zone" to activate triceps by way of the corticospinal tracts are 5 and $12 \mathrm{msec}$, respectively, with a mode at 5 to 6 msec.

\section{Discussion}
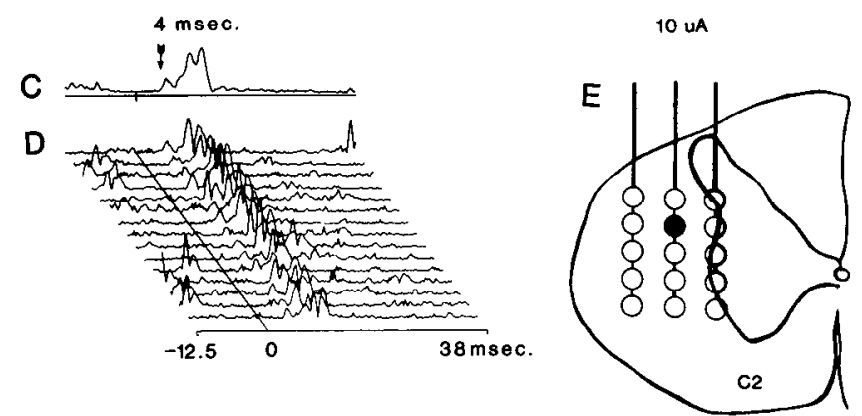

Figure 9. Afferent and efferent "loop time" measurements. $A$ and $B$, Orthodromic and antidromic latencies of $\mathrm{MCNs}$ responding to forelimb displacements. $A$, Afferent response latencies measured from the initiation of the torque motor pulse to the initial point at which the average response histogram peak crosses the $3 \mathrm{SD}$ level (cf. Fig. $8 D$ ). $U$, unresponsive neurons on the same electrode tracks in the "receiving" zone where excited MCNs were recorded (i.e., only a subpopulation of MCNs in the zone showed responses of initial excitation, while others were initially inhibited or unrelated to forelimb displacement). $B$, Antidromic latencies of MCNs to intraspinal microstimulation at $\mathrm{C} 2 . C$ to $E$, Example of orthodromic response in triceps brachii to intraspinal microstimulation at a C2 location where MCNs in the "receiving" zone could be antidromically activated. Average triceps EMG response $(C)$ and sample rasters $(D)$ from which it was constructed. Zero time $=$ stimulus onset. $E$, Reconstruction of penetration grid at the level of $\mathrm{C} 2$. Open circles indicate no EMG response at 10 $\mu \mathrm{A}$; solid circle indicates the locus from which the responses shown in $C$ and $D$ were evoked.

latencies of MCNs in the "receiving zone" were measured following intraspinal microstimulation in the dorsolateral funiculus at $\mathrm{C} 2$ or $\mathrm{C} 3$ (Fig. $9 E$ ). MCNs conforming to the criteria for antidromic invasion (see "Materials and Methods") showed latencies ranging from 1 to 7 msec with a major mode between 1 and 2 msec (Fig. $9 B$ ).

Microstimulation at the same spinal loci that antidromically activated MCNs in the "receiving zone" could evoke EMG responses in triceps brachii at latencies of 4 to 5 msec as illustrated in Figure $9 C$ and $D$. The regions of the spinal cord from which both EMG activity and antidromic activation could be evoked were highly localized, moving the electrode 1 to $2 \mathrm{~mm}$, resulting in complete loss of the EMG response (note that the negative stimulation zones in the schematic drawing of Fig. $9 E$ are only $1 \mathrm{~mm}$ away from the "active" site). The responses in triceps obtained in this manner were bimodal, the early component having the lower threshold. Thus it

\section{Homologies and disparities in segmented EMG responses to sudden displacements}

The EMG segments reported here appear to correspond well with those reported by Ghez and Shinoda (1978) in cat triceps. First, the reported latencies of the three peaks are in agreement with those obtained here at the highest step load values imposed from initial joint angles of less than 100 to $110^{\circ}$. Second, the "gain" curves over identical input load values are at least qualitatively comparable in the two studies for peaks 1 and 2 (see Fig. 2 in Ghez and Shinoda, 1978). Finally, the presence of a "silent interval" is common to both studies (although this phenomenon was not commented on by Ghez and Shinoda (1978), it is evident in a number of their figures). However, the fact that these three peaks are not invariably present, and only occur over a specific range of velocities and initial joint angles, was not previously reported.

Ghez and Shinoda (1978) equated the second and third peaks evoked in cat triceps with the functional stretch reflex in the human ankle (Melvill Jones and Watt, 1971) and with the M2 and M3 of the monkey upper limb (Tatton et al., 1975), which had been proposed as possible expressions of a transcortical reflex functioning to counteract unexpected loads on the joint-muscle system (Phillips, 1969; Marsden et al., 1972). Since the human and monkey wrist muscles have been extensively investigated with torque motor paradigms, they will serve here as a basis for comparison with the cat triceps.

Peak 1. The first observation of note when the two systems are compared is that the cat triceps peak 1 occurred at a shorter latency ( 8 to $12 \mathrm{msec}$ ) than the monkey wrist musculature's M1 (13 to $16 \mathrm{msec})$, consistent with the shorter peripheral and central conduction pathways in the smaller cats. Peak 1 was of constant latency and duration at all initial joint angles and load magnitudes. Its amplitude modulated with initial joint angle and increased with average velocity of displacement, saturating at higher velocities, indicating that the range of velocities used in these experiments included the dynamic range of this response. The response properties of peak 1 in the cat triceps are therefore compatible with those of a segmental reflex. The early components reported for other muscle-joint systems (see Jaeger et al., 1982, for a review) show similar characteristics, and therefore it is possible, but not necessary, that these components are homologous between species and musclejoint systems.

Silent interval. A "silent interval," when EMG activity 
fell below the prestimulus background levels, was interjected between peaks 1 and 2 in cat triceps. This interval is also present in the decerebrate or spinal cat (see Figs. 6 and 7 in Ghez and Shinoda, 1978). It does not appear in primate wrist muscles, where M2 is contiguous with and arises out of the falling phase of the M1 segment in averaged data (compare schematic responses in the lower panels of Fig. 10).

The relationship between the silent interval and the prior appearance of peak 1 is not a simple one. Although the conditions necessary for the expression of the silent interval are shared with peak 1 (if peak 1 is not expressed, neither is the silent interval), there is no clear-cut dependence of silent interval duration on the amplitude of the immediately preceding peak 1 . Alternately, the du-

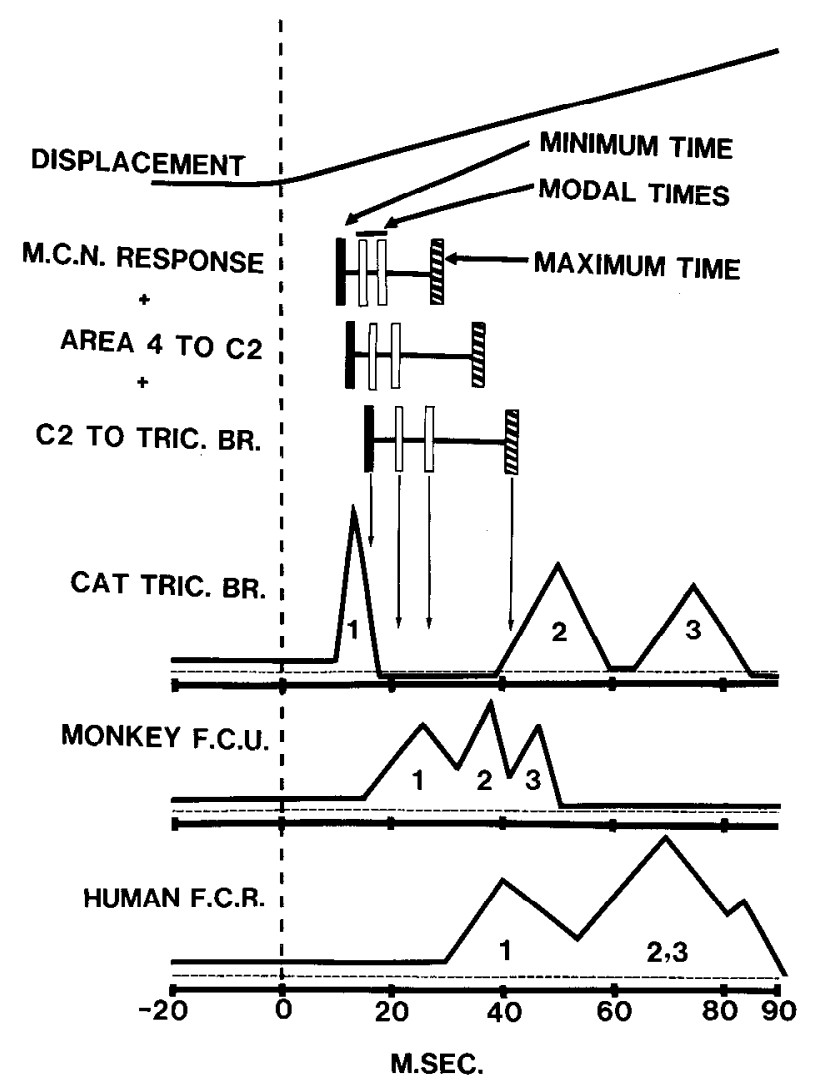

Figure 10. Summary diagram illustrating the findings obtained from the cat triceps and comparison with monkey and man. All traces are plotted relative to time on the abscissa, where 0 indicates the onset of a torque pulse. DISPLACE$M E N T$ indicates the angular joint rotation produced by the motor. The vertical bars show the minimum (solid bars), modal (open bars), and maximum (hatched bars) times at which the imposed movement evokes an M.C.N. RESPONSE, the conduction time from $A R E A 4$ to $C 2$ derived from antidromic activation, and the conduction time from C2 TO TRIC. BR. (triceps brachii) measured by intraspinal stimulation. The vertical arrows indicate the minimum, modal, and maximum times at which a transcortical reflex loop would be expected to evoke EMG activity in the cat. The actual EMG responses are schematized for CAT TRIC. BR and, to highlight the differences, schematic responses for MONKEY F.C.U. (flexor carpi ulnaris; drawn from Bawa and Tatton, 1979) and HUMAN F.C.R. (flexor carpi radialis; drawn from Bedingham and Tatton, 1981; Lee and Tatton, 1982) are illustrated on the same time scale. See the text for further details. ration of the silent interval is monotonically related to the average velocity of displacement, usually becoming asymptotic to a minimal value of about $5 \mathrm{msec}$ at average velocities greater than $300^{\circ} / \mathrm{sec}$. The presence of a silent interval thus clearly distinguishes responses in cat triceps from those at the primate wrist.

Peak 2. In contrast to the findings for peak 1 , it is striking that the cat peak 2 has a longer latency (34 to 52 msec, depending on the torque applied and the initial joint angle; see also Fig. 2 in Ghez and Shinoda (1978), where "M2" latency decreases from 50 to $40 \mathrm{msec}$ ) than that of the macaque M2 (32 to $36 \mathrm{msec}$ ) under similar input conditions (Bawa and Tatton, 1979). Importantly, the latency of peak 2 is determined by the duration of the "silent interval."

The influence of initial joint angle seen in the cat is not found in the primate wrist, where M2 latency is independent of initial joint angle if background activity is controlled for (Bedingham, 1981; W. G. Tatton and P. Bawa, unpublished observations). Furthermore, in view of the relatively constant latencies of modal MCN responses (3 msec range of dispersion), a similar constancy might be expected in EMG dependent on the motor cortex. This condition appears to be satisfied in the case of the human or monkey wrist M2, where $\pm 2 \mathrm{msec}$ variation occurs in a given subject, irrespective of the input so long as it exceeds some threshold level (Lee and Tatton, 1982; Lenz et al., 1983a). But peak 2 in cat triceps shows a wide range of latencies $(20 \mathrm{msec})$ in a single animal, depending upon the input conditions. Therefore, peak 2 presents several specific disparities and is unlikely to be homologous with the functional stretch reflex (Chan et al., 1979), M2-M3 (Tatton et al., 1975), ML-LL (O'Riain et al., 1979), or late MR (Jaeger et al., 1982).

Peak 3. Peak 3 and later peaks or the tonic activity which may occur are not clearly related to the step load input. Peak 3 is also present in the spinal cat (Ghez and Shinoda, 1978), but seems to modulate only to a limited extent with different loads. It differs from the M3 of the monkey or human wrist in that peak 3 is consistently expressed only at high loads and shows as a discrete burst of EMG activity following peak 2. Most often in human subjects, the M3 is not discernible (see Fig. 10, human $F$. C. $R$. (flexor carpi radialis)), whereas in the monkey, M3 may often fail to occur, but when present arises from the falling phase of M2 (see Fig. 10, monkey F. C. U. (flexor carpi ulnaris)). Indeed, the EMG activity following perturbations at the primate wrist is hest considered as comprising two major segments which have been termed M1 and M2-3 (see bottom panel in Fig. 10; Lee and Tatton, 1982). The paradigm used in the present experiments does not allows us to draw any conclusions as to the relationship between peak 3 and the "postmyotatic" response observed in man (Jaeger et al., 1982).

\section{Homologies and disparities in temporal relations between $M C N$ and EMG activity}

In previous estimates of "loop times" through the motor cortex, we were limited to citing minimum times for afferent and efferent linkages (e.g., Tatton and Bruce, 1976). The present "loop analysis" method allows us to 
include a major proportion of possible times by using extreme range values and modal values for both the afferent (MCNs which may receive inputs from a variety of receptors via a number of pathways) and efferent (the majority of corticospinal fibers are included in the antidromically identified population with conduction times of $7 \mathrm{msec}$ or less) limbs of the loop. We have made the assumption that the responding MCNs make up a proportion of the population identified by antidromic stimulation, since the same cortical volume was sampled in both cases. The study could have been extended by chronic implantation of electrodes in the pyramidal tracts, but it was recognized that the distribution of antidromic times for MCNs identified as projecting to the pyramids could be altered by the damage incurred during movements of the brainstem.

The latencies of the excitatory $\mathrm{MCN}$ responses to imposed displacements ranged between 10 and $28 \mathrm{msec}$, the range for antidromic conduction times from $\mathrm{C} 2$ was 1 to $7 \mathrm{msec}$, and the time from $\mathrm{C} 2$ to triceps activation was 4 to 5 msec. Thus, the minimum latency from step load onset at which a pathway traversing the motor cortex and descending in the corticospinal tract could alter triceps EMG activity is $15 \mathrm{msec}$. This would place the EMG activity on the falling phase of peak 1 (solid bars in Fig. 10) and, therefore, MCN activity could contribute to the "silent interval." Similarly, the maximum latency sums to $40 \mathrm{msec}$, barely enough time for descending activity to contribute to peak 2 (striped bars in Fig. 10). However, these are extreme values derived from a small proportion of the MCNs actually encountered, and consideration of the modal values provides a more representative picture.

Well defined modes (containing $33 \%$ and $22 \%$ of the responding neurons) were found for the excitatory response latencies of MCNs at 14 to 16 and 18 to $20 \mathrm{msec}$, and a single mode (containing $41 \%$ of activated MCNs) at 1 to 2 msec was found for antidromic conduction times from $\mathrm{C} 2$. (It was considered important to stimulate in the area of $\mathrm{C} 2$ because the corticospinal linkages to forelimb propriospinal neurons occur in this region (Grant et al., 1980).) Using the same summing method as above, we obtain values of 19 to $23 \mathrm{msec}$ and 23 to 27 msec as the most probable times at which the majority of MCNs in the receiving zone would influence EMG activity recorded in triceps brachii (open bars in Fig. 10). Taking these values, any activity contributed by a transcortical loop would occur during the first half of the "silent interval," possibly during the falling phase of peak 1 , but far too early to have any influence on peak 2, which Ghez and Shinoda (1978) homologized with the M2 at the monkey wrist (see their Fig. 1). Recently, further support has been provided for the differences in cat and monkey "loop times" by E. E. Fetz and P. D. Cheney (personal communication) who showed, by the method of spike-triggered facilitation, that MCNs responding to perturbations facilitate EMG activity at the appropriate latency to contribute to the M2 segment in the monkey.

\section{Conclusions}

In marked contrast to peak 2 in cat triceps, the second component of the EMG response at the monkey wrist appears to be appropriately timed to be influenced by activity in MCNs (schematized in Fig. 10, monkey F.C.U.)) as previously pointed out (Evarts, 1973; Tatton and Bruce, 1976; Tatton et al., 1978) and demonstrated with a different "loop analysis" technique by Chofflon et al. (1982). 'Two limitations could be argued in regard to the present study: (1) since they were measured in two different experiments, the responding MCNs and antidromically identified MCNs may represent different populations, and (2) corticospinal neurons with antidromic conduction times greater than $7 \mathrm{msec}$ (conduction velocities of 5 to $10 \mathrm{~m} / \mathrm{sec}$ ) may be involved. Nevertheless, this approach has significantly improved over the previous studies and, furthermore, analysis such as that reported here, carried out in other species and jointmuscle systems, may provide further explanations of the conflicting reports of presence or absence of long latency (M2-3) EMG segments following lesions which would be expected to interrupt a transcortical pathway in primates.

It is noteworthy that most of the studies which showed attenuated or abolished M2 peaks following motor cortical lesions were carried out in the distal limb of primates (e.g., Marsden et al., 1977; Lee and Tatton, 1978), whereas those which reported no lesion effect utilized the proximal limb of monkeys (Tracey et al., 1980; Miller and Brooks, 1981) or cats (Ghez and Shinoda, 1978). Such proximodistal differences have recently been analzyed in the squirrel monkey forelimb, where a motor cortical lesion eliminated or decreased M2 activity in distal flexors, but not in proximal flexor muscles (Lenz et al., 1983b), in parallel with the finding that the predominance of the M2 segment in the normal limb increased from proximal to distal musculature (Lenz et al., 1983a). A clear understanding of the physiological basis of these responses is of crucial importance for: (1) the choice of an appropriate species for investigating a given aspect of motor control, (2) defining the pathophysiological events leading to movement disorders in man, and (3) quantitating the effectiveness of therapeutic regimens designed to ameliorate such disorders.

To conclude, in our view the present study underlines the fact that Phillips' (1969) original proposal of a transcortical reflex system was based upon studies of the monosynaptic cortico-motoneuronal connections for the distal upper limb musculature of primates and, consequently, studies showing the persistence of EMG segmentation in other species and/or joint-muscle systems following lesions which might be expected to interrupt the loop cannot be taken as adequate evidence to refute or to confirm that hypothesis.

\section{References}

Alstermark, B., A. Lundberg, U. Norsell, and E. Sybirska (1981) Integration in descending motor pathways controlling the forelimb in the cat. IX. Differential behavioural defects after spinal cord lesions interrupting defined pathways from higher centres to motoneurones. Exp. Brain Res. 42: 299-318.

Bawa, P., and W. G. Tatton (1979) Motor unit responses in muscles stretched by imposed displacements of the monkey wrist. Exp. Brain Res. 37: 417-437.

Bedingham, W. (1981) Input:output properties of the human wrist reflex-A model for studying neuromotor diseases. M.Sc. thesis, University of Toronto, Ontario, Canada. 
Redingham, W., and W. G. Tatton (1981) Characteristic longlatency reflex abnormalities in dystonia and rigidity. Can. Congr. Neurol. Sci. 13: 138.

Bruce, I. C., and W. G. Tatton (1980) Sequential output-input maturation of kitten motor cortex. Exp. Brain Res. 39: 411419.

Chan, C. W. Y., R. E. Kearney, and G. Melvill Jones (1979) Tibialis anterior response to sudden ankle displacements in normal and Parkinsonian subjects. Brain Res. 173: 303-314.

Chofflon, M., J. M. Lachat, and D. G. Ruegg (1982) A transcortical loop demonstrated by stimulation of low-threshold muscle afferents in the awake monkey. J. Physiol. (Lond.) 323: 393-402.

Evarts, E. V. (1973) Motor cortex reflexes associated with learned movements. Science 179: 501-503.

Ghez, C., and Y. Shinoda (1978) Spinal mechanisms of the functional stretch reflex. Exp. Brain Res. 32: 55-68.

Gottlieb, G. L., and G. C. Agarwal (1980) Response to sudden torques about ankle in man. II. Postmyotatic reactions. J. Neurophysiol. 43: 86-101.

Grant, G., M. Illert, and R. Tanaka (1980) Integration in descending motor pathways controlling the forelimb in the cat. VI. Anatomical evidence consistent with the existence of C3-C4 propriospinal neurons projecting to forelimb motor nuclei. Exp. Brain Res. 39: 87-93.

Hammond, P. H. (1954) Involuntary activity in biceps following the sudden application of velocity to the abducted forearm. J. Physiol. (Lond.) 127: 17P-18P.

Hassler, R., and K. Muhs-Clement (1964) Architektonischer Aufbau der Sensomotorischen und Parietalen Cortex der Katze. J. Hirnforsch. 6: 377-420.

Jaeger, R. J., G. L. Gottlieb, and G. C. Agarwal (1982) Myoelectric responses at flexors and extensors of human wrist to step torque perturbations. J. Neurophysiol. 48: 388-402.

Lee, R. G., and W. G. Tatton (1978) Long loop reflexes in man: Clinical applications. In Cerebral Motor Control in Man: Long Loop Mechanisms, J. E. Desmedt, ed., pp. 320-333, S. Karger AG, Basel, Switzerland.

Lee, R. G., and W. G. Tatton (1982) I ong latency reflexes to imposed displacements of the human wrist: Dependence on duration of movement. Exp. Brain Res. 45: 207-216.

Lenz, F. A., W. G. Tatton, and R. R. Tasker (1983a) Electromyographic response to displacement of different forelimb joints in the squirrel monkey. J. Neurosci. 3: 783-794.

Lenz, F. A., W. G. Tatton, and R. R. Tasker (1983b) The effect of cortical lesions on the electromyographic response to joint displacement in the squirrel monkey forelimb. J. Neurosci. 3: 795-805.

Marsden, C. D., P. E. Merton, and H. B. Morton (1972) Servo action in human voluntary movement. Nature (Lond.) 238: 140-143.

Marsden, C. D., P. E. Merton, H. B. Morton, and J. Adam (1977) The effect of lesions of sensorimotor cortex and the capsular pathways on servo responses from the human long thumb flexor. Brain 100: 185-200.

Melvill Jones, G., and D. G. D. Watt (1971) Observations on the control of stepping and hopping movements in man. J. Physiol. (Lond.) 219: 709-727.

Miller, A. D., and V. B. Brooks (1981) Late muscular responses to arm perturbations persist during supraspinal dysfunctions in monkeys. Exp. Brain Res. 41: 146-158.

North, A. G. E., and W. G. Tatton (1980) Temporal relation between motor cortical and electromyographic responses to imposed forelimb displacements in the cat. Soc. Neurosci. Abstr. 6: 392.

O'Riain, M. D., R. D. G. Blair, and J. T. Murphy (1979) Modifiability of the stretch reflex by prior information. Electromyogr. Clin. Neurophysiol. 19: 57-63.

Phillips, C. G. (1969) Motor apparatus of the baboon's hand. Proc. R. Soc. Lond. Biol. 173: 141-174.

Tatton, W. G., and P. Bawa (1979) Input-output properties of motor unit responses in muscles stretched by imposed displacements of the monkey wrist. Exp. Brain Res. 37: 439457.

Tatton, W. G., and I. C. Bruce (1976) Temporal relations between motor cortical neuronal responses and EMG responses to sudden load changes applied to the primate wrist. Can. J. Physiol. 7: 59.

Tatton, W. G., S. D. Forner, G. L. Gerstein, W. W. Chambers, and C. M. Liu (1975) The effect of postcentral cortical lesions on motor responses to sudden upper limb displacements in monkeys. Brain Res. 96: 108-113.

Tatton, W. G., P. Bawa, I. C. Bruce, and R. G. Lee (1978) Long-loop reflexes in monkeys: An interpretative base for human reflexes. In Cerebral Motor Control in Man: Long Loop Mechanisms, J. E. Desmedt, ed., pp. 229-245, S. Karger AG, Basel, Switzerland.

Tracey, D. J., B. Walmsley, and J. Brinkman (1980) "Long loop" reflexes can be obtained in spinal monkeys. Neurosci. Lett. 18: 59-66. 